\title{
The Impact of GFP Reporter Gene Transduction and Expression on Metabolomics of Placental Mesenchymal Stem Cells Determined by UHPLC-Q/TOF-MS
}

\author{
Jinfeng Yang, ${ }^{1,2}$ Nan Wang, ${ }^{2,3}$ Deying Chen,, Jiong Yu, ${ }^{1,2}$ Qiaoling Pan,,2 Dan Wang, ${ }^{1,2}$ \\ Jingqi Liu, ${ }^{1,2}$ Xiaowei Shi, ${ }^{4}$ Xiaotian Dong, ${ }^{1,2}$ Hongcui Cao, ${ }^{1,2}$ Liang $\mathrm{Li}^{2,3}$ and Lanjuan $\mathrm{Li}^{1,2}$ \\ ${ }^{1}$ State Key Laboratory for the Diagnosis and Treatment of Infectious Diseases, The First Affiliated Hospital, College of Medicine, \\ Zhejiang University, Hangzhou, China \\ ${ }^{2}$ Collaborative Innovation Center for the Diagnosis and Treatment of Infectious Diseases, Zhejiang University, \\ Hangzhou 310003, China \\ ${ }^{3}$ Department of Chemistry, University of Alberta, Edmonton, AB, Canada T6G 2 G2 \\ ${ }^{4}$ Chu Kochen Honors College, Zhejiang University, 866 Yuhangtang Rd., Hangzhou 310058, China
}

Correspondence should be addressed to Hongcui Cao; hccao@zju.edu.cn

Received 24 April 2017; Revised 23 July 2017; Accepted 7 August 2017; Published 5 November 2017

Academic Editor: Yonglun Luo

Copyright (c) 2017 Jinfeng Yang et al. This is an open access article distributed under the Creative Commons Attribution License, which permits unrestricted use, distribution, and reproduction in any medium, provided the original work is properly cited.

\begin{abstract}
Introduction. Green fluorescent protein (GFP) is widely used as a reporter gene in regenerative medicine research to label and track stem cells. Here, we examined whether expressing GFP gene may impact the metabolism of human placental mesenchymal stem cells (hPMSCs). Methods. The GFP gene was transduced into hPMSCs using lentiviral-based infection to establish $\mathrm{GFP}^{+} \mathrm{hPMSC}$. A sensitive ${ }^{13} \mathrm{C} /{ }^{12} \mathrm{C}$-dansyl labeling LC-MS method targeting the amine/phenol submetabolome was used for indepth cell metabolome profiling. Results. A total of 1151 peak pairs or metabolites were detected from 12 LC-MS runs. Principal component analysis and partial least squares discriminant analysis showed poor separation, and the volcano plots demonstrated that most of the metabolites were not significantly changed when hPMSCs were tagged with GFP. Overall, 739 metabolites were positively or putatively identified. Only 11 metabolites showed significant changes. Metabolic pathway analyses indicated that three of the identified metabolites were involved in nine pathways. However, these metabolites are unlikely to have a large impact on the metabolic pathways due to their nonessential roles and limited hits in pathway analysis. Conclusion. This study indicated that the expression of ectopic GFP reporter gene did not significantly alter the metabolomics pathways covered by the amine/phenol submetabolome.
\end{abstract}

\section{Introduction}

Mesenchymal stem cells (MSCs) are the current focus for the development of cell-based therapies for various diseases due to their regenerative and immune regulatory potential $[1,2]$. For the preclinical application of MSCs, labeling and tracking are crucial for evaluating the in vivo distribution and fate of transplanted stem cells over time. Green fluorescent protein (GFP) has been used to evaluate cellular engraftment and for long-term cell tracking [3, 4]. Our previous studies showed that transfection of human placental mesenchymal stem cells (hPMSCs) with the GFP gene did not affect their viability, phenotype profile, or pluripotency [5]. However, the impact of GFP labeling on the metabolism of MSCs remains unknown. Metabolomics provides a unique platform for the systematic study of small molecule metabolites from biological samples by allowing the simultaneous assessment of large numbers of metabolites. Metabolomics has been widely applied in various research fields, including biomarker discovery, drug 
discovery, disease diagnosis, and other biology-related fields $[6,7]$. In the context of regenerative medicine, metabolomics analysis of cells has become an important technique in the study of cell biochemistry because large numbers of chemicals can be quantified to provide detailed insight into the metabolic status of cells $[8,9]$.

Here, we report the development and application of an effective metabolite extraction protocol, along with a highly sensitive metabolomic profiling method based on chemical isotope labeling liquid chromatography-mass spectrometry (CIL LC-MS) to investigate whether GFP gene transduction and expression impacts the metabolism of hPMSCs. High coverage of metabolites was achieved, which allowed better understanding of the potential cellular disturbances induced by GFP labeling.

\section{Materials and Methods}

2.1. GFP Gene Transduction. All protocols for human tissue and cell handling were approved by the Research Ethics Committee of the First Affiliated Hospital, School of Medicine, Zhejiang University (reference number 2013272). The culture and labeling of hPMSCs were performed according to previously reported protocols $[5,10]$. The GFP gene was transduced into hPMSCs with a lentivirus to establish $\mathrm{GFP}^{+}$hPMSCs. The lentivirus-based short hairpin RNA (shRNA) vector, pGLV3/H1/GFP ${ }^{+}$Puro, containing nonspecific shRNA driven by the $\mathrm{H} 1$ promoter containing GFP and puromycin, was purchased from GenePharma Inc. (Shanghai, China). The vectors were integrated and replicated in Escherichia coli (Sangon Biotech Corp., Shanghai, China) and purified using a plasmid preparation kit (Qiagen, Hilden, Germany). 293 T cells (Sangon Biotech Corp.) were separately transfected with this plasmid and three packaging plasmids (pGag/Pol, pRev, pVSV-G) using Lipofectamine 2000 (Thermo Fisher Scientific Inc., Waltham, MA, USA) according to the manufacturer's instructions and cultured in the presence of G418 (400 mg/L; Sigma-Aldrich Co., St. Louis, MO, USA). The lentivirus supernatant was collected after $293 \mathrm{~T}$ cells reached $80 \%$ confluence and stored at $-80^{\circ} \mathrm{C}$ for future use; the titer was determined by GFP expression assay. hPMSCs were seeded and cultured in six-well plates at a density of $1 \times 10^{5} /$ well for $24 \mathrm{~h}$ in special medium (Mesen Cult ${ }^{\circledR}$ Human Basal Medium plus MesenCult ${ }^{\circledR}$ Human Supplement; STEMCELL Technologies Inc., Vancouver, $\mathrm{BC}$, Canada). On the day of transduction, $2 \mathrm{~mL}$ of fresh culture medium containing $5 \mu \mathrm{g} / \mathrm{mL}$ polybrene and lentiviral vectors was added to each well. After $24 \mathrm{~h}$, the medium was then replaced with fresh medium containing $10 \mu \mathrm{g} / \mathrm{mL}$ puromycin (Sigma-Aldrich). The puromycin-resistant cells were observed by a fluorescence phase contrast microscope (IX2-UCB-2; Olympus, Tokyo, Japan) and collected to produce a pool of hPMSCs.

2.2. Surface Antigen Detection. $\mathrm{GFP}^{+}$hPMSCs (passages 2-5) were collected and incubated in the dark with allophycocyanin-labeled antibodies against CD34, CD45, CD73, CD90, and CD105 (eBioscience, San Diego, CA, USA) for about $30 \mathrm{~min}$. The cells were washed with phosphate-buffered saline and then examined using a flow cytometry (Cytomics FC 500 MPL; Beckman Coulter, Brea, CA, USA).

2.3. Metabolite Extraction. Metabolite extraction was performed according to the protocols described by Lorenz et al. $[11,12]$ with some modifications. Briefly, hPMSCs and $\mathrm{GFP}^{+}$hPMSCs at passages 3-5 were seeded on T175 tissue culture flasks (Nunc ${ }^{\mathrm{TM}}$ EasYFlasks ${ }^{\mathrm{TM}}$; Thermo Fisher Scientific Inc.) and cultured to $80-90 \%$ confluence. The metabolites were extracted from the cells using the following procedure: cells were removed from the media, rapidly rinsed using approximately $20 \mathrm{~mL}$ of $37^{\circ} \mathrm{C}$ ultrapure water, and quenched within $5 \mathrm{~s}$ by directly adding approximately $15 \mathrm{~mL}$ of liquid nitrogen $\left(\mathrm{LN}_{2}\right)$ to the flask. For extraction method 1, $3 \mathrm{~mL}$ of ice-cold 9/1 $(v / v)$ methanol $(\mathrm{MeOH}) /$ chloroform $\left(\mathrm{CHCl}_{3}\right)$ (Fisher Chemical, Waltham, MA, USA) was immediately added to the flask. For extraction method 2, 1/1 $(v / v) \mathrm{MeOH} / \mathrm{H}_{2} \mathrm{O}\left(-20^{\circ} \mathrm{C}, 3 \mathrm{~mL}\right)$ was directly added to the culture flask after the washing procedure. The adherent cells were then scraped from the culture flasks using cell scrapers (B-1420; Orange Scientific, Braine-l'Alleud, Belgium). The solutions were transferred to $15 \mathrm{~mL}$ centrifuge tubes (Thermo Fisher Scientific Inc.), ultrasonicated for $30 \mathrm{~s}$ (KH-250B; Kunshan, Jiangsu, China), and then transferred to $1.5 \mathrm{~mL}$ microcentrifuge tubes and spun down at $4^{\circ} \mathrm{C}$ for $10 \mathrm{~min}$ at $16100 \times \mathrm{g}$ (Microfuge 22R; Beckman Coulter). The supernatants were dried using a refrigerated CentriVap concentrator system (Labconco, Kansas City, MO, USA), resuspended in $\mathrm{H}_{2} \mathrm{O}$, and centrifuged at $4^{\circ} \mathrm{C}$ for $10 \mathrm{~min}$ at $16100 \times \mathrm{g}$. The supernatants were subsequently transferred to new tubes for labeling.

2.4. Derivatization, Normalization, and Mixing. Dansylation labeling was performed according to the protocol reported previously [12]. The ${ }^{12} \mathrm{C}$-dansyl chloride (light chain) was purchased from Sigma-Aldrich, and ${ }^{13} \mathrm{C}$-dansyl chloride (heavy chain) was synthesized in-house as previously described [12]. The individual samples were separately labeled by ${ }^{12} \mathrm{C}$-dansylation or ${ }^{13} \mathrm{C}$-dansylation. In brief, $50 \mu \mathrm{L}$ of the cell extract was mixed with $25 \mu \mathrm{L}$ of sodium carbonate/sodium bicarbonate buffer $(0.5 \mathrm{~mol} / \mathrm{L}, \mathrm{pH} 9.5$; Sigma-Aldrich) and $25 \mu \mathrm{L}$ of acetonitrile (CAN; SigmaAldrich) in a screw-capped vial. The vial was vortexed and centrifuged. Then, $50 \mu \mathrm{L}$ of freshly prepared ${ }^{12} \mathrm{C}$-dansyl chloride or ${ }^{13} \mathrm{C}$-dansyl chloride in ACN $(20 \mathrm{mg} / \mathrm{mL})$ was added for light or heavy labeling, respectively. The solution was vortexed and centrifuged again. The dansylation reactions were performed in a water bath at $60^{\circ} \mathrm{C}$ for $60 \mathrm{~min}$, after which $10 \mu \mathrm{L}$ of sodium hydroxide solution $(250 \mathrm{mM})$ was added to quench the excess dansyl chloride. After an additional $10 \mathrm{~min}$ incubation $\left(60^{\circ} \mathrm{C}\right), 50 \mu \mathrm{L}$ of $425 \mathrm{mM}$ formic acid in $\mathrm{ACN} / \mathrm{H}_{2} \mathrm{O}(1: 1, v / v)$ was added to neutralize the solution. This solution was diluted by adding $10 \% \mathrm{ACN} / 0.1 \%$ formic acid (FA; Sigma-Aldrich) at a ratio of $1: 1(v / v)$. The ${ }^{12} \mathrm{C}$-dansylated samples were then mixed with the corresponding ${ }^{13} \mathrm{C}$-dansylated sample from the same extraction method but using the other cell type in a molar ratio of $1: 1$. 
After labeling with ${ }^{12} \mathrm{C} /{ }^{13} \mathrm{C}$-dansyl chloride, the labeled metabolites were quantified by LC-UV [13] to control the amount of sample used for metabolome comparison. An ultra-high performance liquid chromatography (UHPLC) system (Agilent 1290; Agilent, Palo Alto, CA, USA) with a photodiode array detector (Agilent) was used for quantification based on the absorption at $338 \mathrm{~nm}$. Briefly, $2 \mu \mathrm{L}$ of the labeled solution was injected into a Waters ACQUITY UPLC BEH C18 analytical column (i.d. $2.1 \times 100 \mathrm{~mm}, 1.7 \mu \mathrm{m}$, pore size $130 \AA$ A ; Waters Co., Milford, MA, USA). LC solvent A consisted of $0.1 \%(v / v)$ formic acid in water, and LC solvent $\mathrm{B}$ consisted of $0.1 \%(v / v)$ formic acid in ACN. The following solvent gradient was used: $15 \%$ B for $1 \mathrm{~min}$, increasing to $98 \%$ B within $0.01 \mathrm{~min}$, holding at $98 \%$ B for $1 \mathrm{~min}$, decreasing to $15 \% \mathrm{~B}$ within $0.5 \mathrm{~min}$, and maintaining this condition for $3.5 \mathrm{~min}$. The flow rate was $500 \mu \mathrm{L} / \mathrm{min}$.

According to the UV quantification results, appropriate volumes of the ${ }^{12} \mathrm{C}$-dansylated samples were then mixed with the corresponding ${ }^{13} \mathrm{C}$-dansylated sample from the same extraction method but using the other cell type in $1: 1$ molar ratios.

2.5. LC-MS. LC-MS was performed using a binary highperformance liquid chromatography system (Agilent 1290 series) connected to an electrospray ionization time-offlight mass spectrometer (Agilent 6230). Chromatographic separation was carried out on a Waters ACQUITY UPLC BEH C18 analytical column $(2.1 \times 100 \mathrm{~mm}, 1.7 \mu \mathrm{m}$, pore size $130 \AA$ A ; Waters Co.); solvent A consisted of water with $0.1 \%$ $(v / v)$ formic acid, and solvent B consisted of ACN with $0.1 \%(v / v)$ formic acid. The gradient was as follows: $t=0 \mathrm{~min}, 15 \% \mathrm{~B} ; t=2 \mathrm{~min}, 15 \% \mathrm{~B} ; t=15 \mathrm{~min}, 45 \% \mathrm{~B}$; $t=20 \mathrm{~min}, 65 \% \mathrm{~B} ; t=26 \mathrm{~min}, 98 \% \mathrm{~B} ; t=29 \mathrm{~min}, 98 \% \mathrm{~B}$; and $t=29.1 \mathrm{~min}, 15 \% \mathrm{~B}$. The flow rate was $250 \mu \mathrm{L} / \mathrm{min}$.

2.6. Data Processing and Statistical Analyses. An R-based inhouse software tool, IsoMS [14], was used to process the raw data generated from the multiple LC-MS runs. A zero-fill program [15] was used to replace a few intensity values that were missing in the LC-MS runs due to low MS sensitivity. Principal component analysis (PCA) and partial least squares discriminant analysis (PLS-DA) were used for multivariate statistical analyses. Volcano plots were prepared using OriginPro version 9.1 (OriginLab, Wellesley Hills, MA, USA). Hierarchical clustering and heat map generation were performed using R version 3.1 (http://www.r-project.org). The metabolites were identified based on retention time and accurate mass matching to a dansyl standard library or accurate mass matching to the human metabolome database (HMDB). The MyCompoundID program (http://www. mycompoundid.org) was used to search for accurate mass matches within the HMDB. The mass accuracy tolerance window was set at $10 \mathrm{ppm}$ for the database search. Metabolite set enrichment analysis and pathway analysis were based on MetaboAnalyst (http://www.metaboanalyst.ca). The Homo sapiens pathway library was used. Cytoscape 3.4 .0 on MetScape (http://www.cytoscape.org) was used for largescale network analysis and the visualization of the integrated metabolism pathways [16].

\section{Results}

3.1. Transduction of hPMSCs with Green Fluorescent Protein. hPMSCs were transduced with a lentiviral vector encoding GFP, and the transduction efficiency was assessed directly by fluorescence and confocal microscopy (LSM 710; Carl Zeiss, Jena, Germany). The vector which yielded transduction efficiency was $>80 \%$ at a multiplicity of infection of $100: 1$. The immunophenotype of $\mathrm{GFP}^{+}$hPMSCs was analyzed by flow cytometry, which indicated that the cells were negative for CD45, CD34, and CD79 but expressed high levels of CD73, CD90, and CD105 (Figure 1). This was consistent with the general description of the phenotypic profile of classical hPMSCs $[5,10]$.

3.2. Workflow. In the overall experimental design, the two types of cells were cultured in triplicate. The metabolites were subsequently extracted using two different extraction solvents. Two aliquots were generated from each of the 12 samples, one of which was labeled with ${ }^{12} \mathrm{C}$-dansyl chloride and the other with ${ }^{13} \mathrm{C}$-dansyl chloride. Each ${ }^{12} \mathrm{C}$-dansylated sample was then mixed with the corresponding ${ }^{13} \mathrm{C}$-dansylated sample derived from the same extraction method but using the other cell type. Overall, 12 mixtures were prepared and individually analyzed by LC-MS.

3.3. Metabolites from Different Extraction Methods. For 9/1 $\mathrm{MeOH} / \mathrm{CHCl}_{3}$ extraction (method 1), the numbers of peak pairs detected were $482,470,505,435,457$, and 435 from the six samples (381 common pairs), and 852, $843,859,867,858$, and 856 were detected for $1 / 1$ $\mathrm{MeOH} / \mathrm{H}_{2} \mathrm{O}$ extraction (method 2) from the six samples (776 common pairs). Online Supplementary Figure S1 available online at https://doi.org/10.1155/2017/3167985 shows a comparison of the peak pairs detected between the two methods, and a larger fraction of the peak pairs was detected in method 2. That is, 327 of a total of 1151 peak pairs detected in all extracts (i.e., 28.41\%) were common, 922 pairs $(80.10 \%)$ were detected in method 2, and 556 pairs $(48.30 \%)$ were detected in method 1 . To obtain a comprehensive list of metabolites, the results from both extraction methods were combined for database search and statistical data analysis.

By searching the 1151 peak pairs against the dansyl standard library, which consists of 273 labeled standards [17], using a mass tolerance of $10 \mathrm{ppm}$ and a retention time (RT) tolerance of $30 \mathrm{~s}, 89$ metabolites were positively identified based on mass and RT matches (see online Supplementary Table S1A for the list). Using MyCompoundID [18] MS search based on the accurate mass of the peak pairs with a mass tolerance of $10 \mathrm{ppm}, 281$ metabolites were putatively identified using the HMDB library (see online Supplementary Table S1B), and 458 metabolites were putatively identified using the predicted human metabolite library (EML) with one reaction (see online Supplementary Table S1C). Thus, of the 1151 peak pairs, a total of 739 metabolites (64.2\%) were positively or putatively identified. The above 


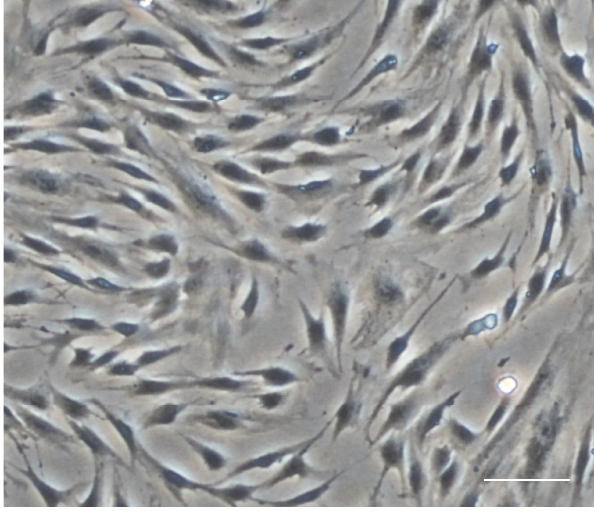

(a)
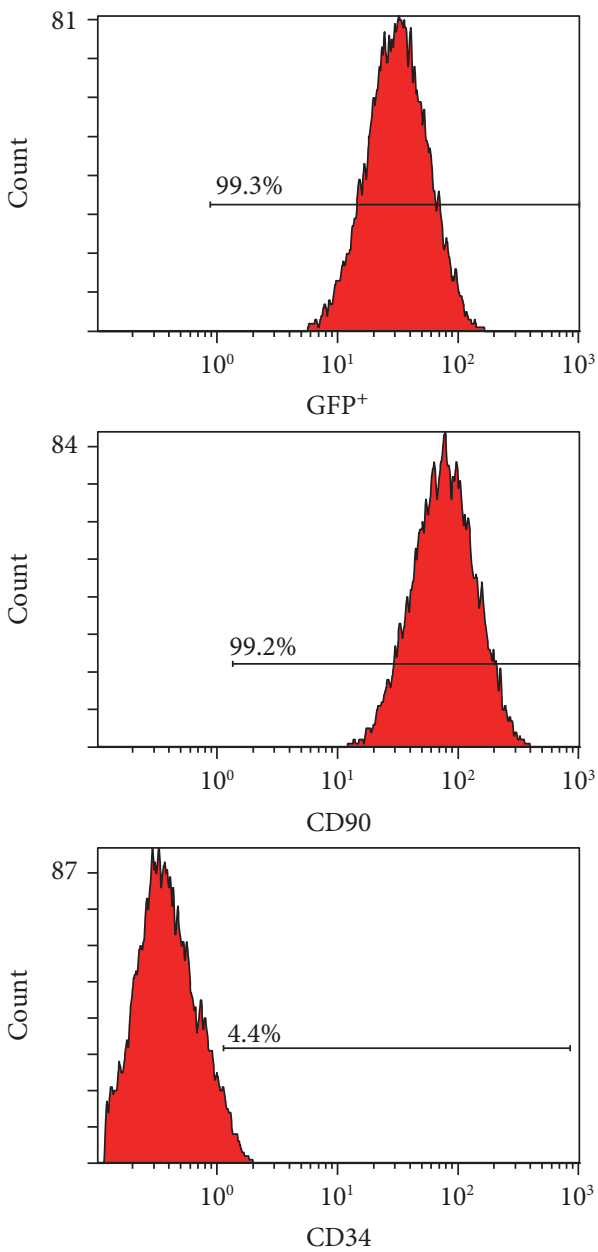

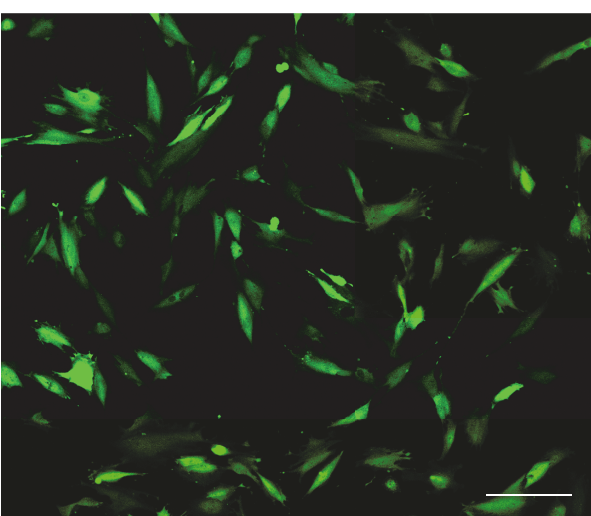

(b)
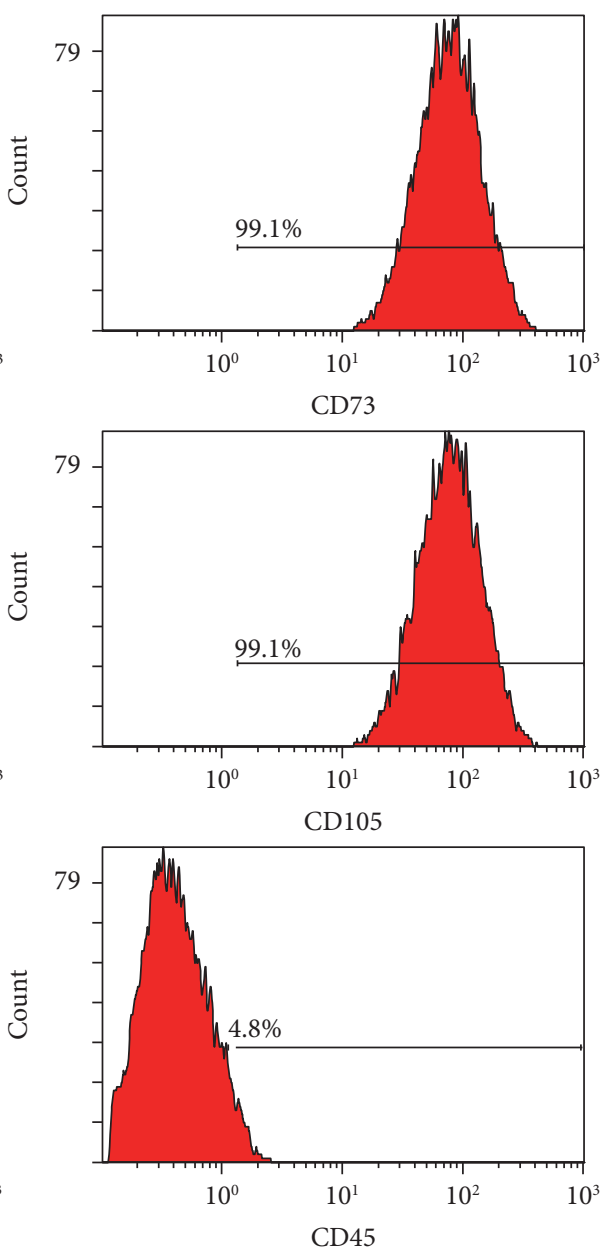

(c)

FIGURE 1: Human placental mesenchymal stem cell (hPMSC) morphology and phenotype profile. (a) hPMSCs without lentiviral transfection. (b) Three days after lentiviral transfection. (c) Surface antigen analysis of green fluorescent protein-positive (GFP ${ }^{+}$) hPMSCs (CD73, CD90, CD105, CD34, and CD45). Scale bars: $100 \mu \mathrm{m}$.

results indicated that dansylation LC-MS can be used to detect and quantify large numbers of metabolites in cell samples.

Hierarchical clustering and heat maps were used to examine the metabolites detected from the two sample processing methods. The normalized intensities of the individual metabolites were used, and the metabolite peaks with similar intensity profiles over the LC-MS runs were clustered together. The clustering results are shown in Figure 2 with deeper red colors representing higher intensities and deeper blue colors representing lower intensities. It is interesting that the color distributions were similar within each type of cells (hPMSCs and $\mathrm{GFP}^{+} \mathrm{hPMSCs}$ ) but were very different 


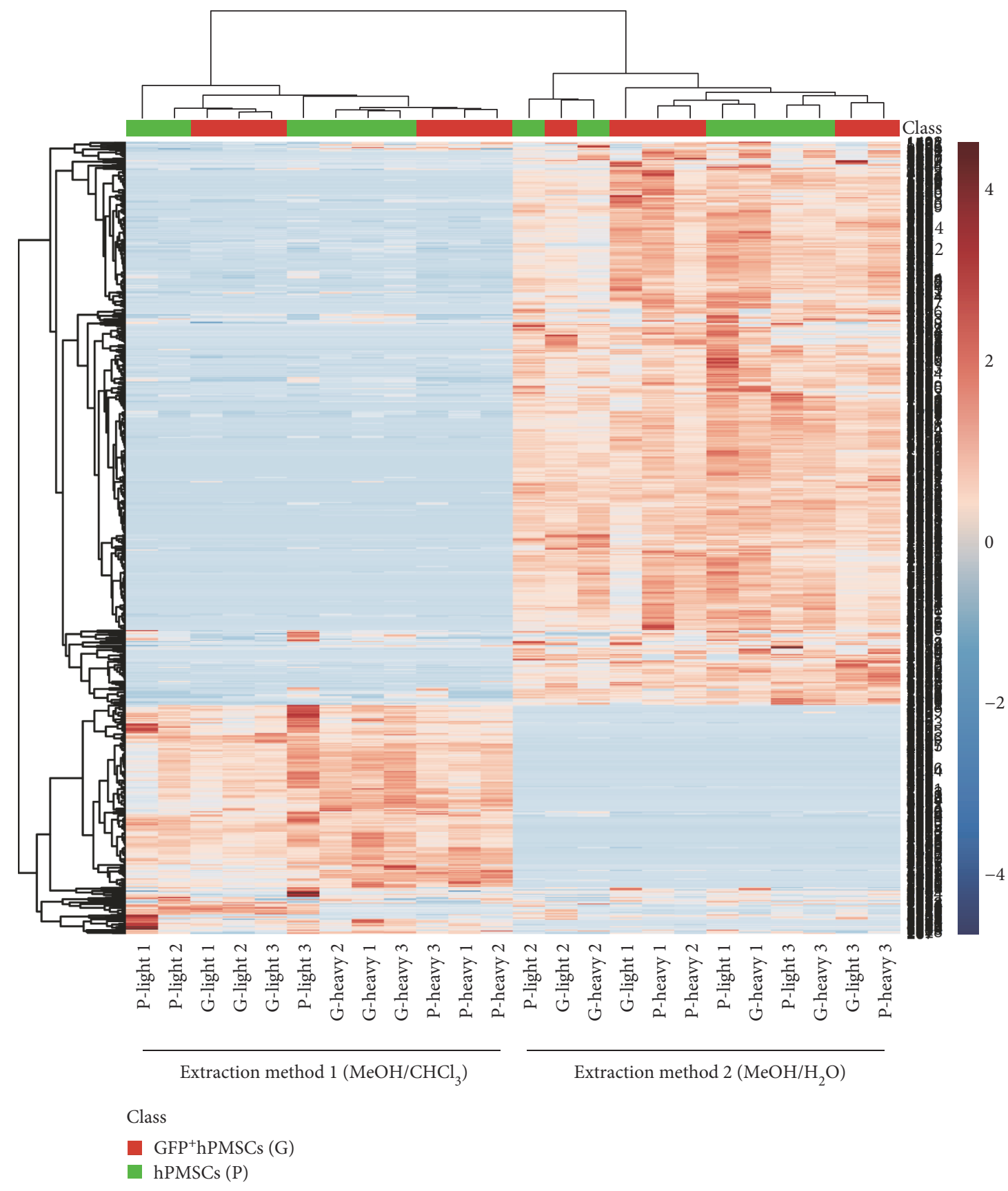

Figure 2: The $9 / 1$ methanol $(\mathrm{MeOH}) /$ chloroform $\left(\mathrm{CHCl}_{3}\right)$ and the $1 / 1 \mathrm{MeOH} / \mathrm{H}_{2} \mathrm{O}$ solvents were used to extract metabolites from the hPMSCs and $\mathrm{GFP}^{+}$hPMSCs. The extracts obtained by the two methods were assayed by positive-ion ultra-high performance liquid chromatography-electrospray ionization ultra-mass spectrometry (UHPLC-ESI-MS). A heat map was generated from the liquid chromatography-mass spectrometry (LC-MS) data by the hierarchical clustering algorithm. The hierarchical clusters were calculated from the individual values using 1 Pearson correlation coefficient as distance and complete linkage for agglomeration. Each data point represents the relative intensity of the metabolite in each sample. The red colors presented in the heat map indicate values above the normalized average value, while blue indicates those below the standard value. The color shade is directly proportional to the intensity.

between the two extraction methods $\left(9 / 1 \mathrm{MeOH} / \mathrm{CHCl}_{3}\right.$ and $1 / 1 \mathrm{MeOH} / \mathrm{H}_{2} \mathrm{O}$ ). The results of the hierarchical clustering analysis indicated that the hPMSCs and the GFP ${ }^{+}$hPMSCs did not show markedly distinct metabolite patterns. In addition, the two different extraction solvents were shown to differ significantly in the types of metabolites extracted, so that the metabolites from these two methods were clustered into two distinct groups. These observations indicated that different extraction solvents had marked effects on the metabolite patterns.

Based on the above results, the $1 / 1 \mathrm{MeOH} / \mathrm{H}_{2} \mathrm{O}$ solvent system provided better extraction, as indicated by the greater number of peak pairs detected and the higher relative peak intensities. However, an integration of the results from 
these two extraction methods improved the overall metabolome coverage.

3.4. Metabolome Profiling. Multivariate statistical analysis was performed using PCA and PLS-DA to examine whether GFP labeling affected the metabolomic profiles of the hPMSCs. Figure 3(a) shows the relevant PCA score scatter plot of the data $\left(R^{2}=0.496, Q^{2}=0.0984\right)$. There was no clear separation between the two groups (hPMSCs and $\mathrm{GFP}^{+}$hPMSCs). The PLS-DA models (Figure 3(b)) also showed poor separation between the two experimental groups $\left(R^{2}=0.987, Q^{2}=0.389\right)$. Volcano plot analyses, which combined the fold change (FC) and the $p$ values from the $t$ tests, were also used to identify the unique metabolites that separated the two groups (hPMSCs and $\mathrm{GFP}^{+} \mathrm{hPMSC}$; Figure 3(c)). The blue dots in the volcano plots represent metabolites that are not statistically significant, whereas the red dots indicate those with significant changes, as defined by their $p$ values and FC. Metabolites with $p<0.05$ and FC either $>1.5$ or $<0.67$ (i.e., $> \pm 50 \%$ change) were considered significant (red dots). Using these thresholds, only $2.3 \%$ (27/1151; Figure 3(c)I) and 1.8\% (21/1151; Figure 3(c)II) of the peak pairs changed significantly in response to GFP transfection.

Using the thresholds of $\mathrm{FC}>2$ or $\mathrm{FC}<0.5$ and $p<0.05$, no peak pairs with significant changes were observed (Figure 3(c)III-IV). The volcano plots indicated that the majority of metabolites did not show dramatic changes when the stem cells were tagged with GFP.

3.5. Metabolite Quantification and Identification. In the present study, we applied a forward-and-reverse labeling strategy to ensure the confidence of the metabolite quantification results and a lower false-positive rate. The ${ }^{12} \mathrm{C}$-dansyl chloride-labeled metabolites extract from the hPMSCs (P) was mixed with the ${ }^{13} \mathrm{C}$-dansyl chloridelabeled metabolites extracted from the $\mathrm{GFP}^{+} \mathrm{hPMSCs}(\mathrm{G})$ (denoted as $\mathrm{G}_{\text {heavy }} \mathrm{P}_{\text {light }}$ ) in a 1:1 molar ratio based on the total metabolite content, as measured by LC-UV. Similarly, the ${ }^{13} \mathrm{C}$-dansyl chloride-labeled hPMSC metabolite extract was mixed with the ${ }^{12} \mathrm{C}$-dansyl chloride-labeled metabolites from GFP ${ }^{+}$hPMSCs (denoted as $\mathrm{G}_{\text {light }} \mathrm{P}_{\text {heavy }}$ ).

Ideally, the relative ratio of a metabolite peak pair determined from the forward labeled mixture would be the reciprocal of the ratio determined from the reverse labeled mixture. Any deviation from the reciprocal relation is mainly attributable to experimental variations. In our analysis, ratios of 1.50 and 0.67 were set as the thresholds for the selection of metabolites with significant changes. The quantification reproducibility, measured as the standard deviation from the mean for a matched pair in the two mixtures, was also taken into account in this case, and a threshold of 0.1 was used. We then narrowed down the 1151 detected metabolites to 11 correlated markers (Table 1), which were significantly changed in response to GFP transfection.

Five of the 11 potential biomarkers were positively identified in HMDB (i.e., taurine, DL-2-aminooctanoic acid, dityrosine, uracil, and gamma-aminobutyric acid). The concentrations of taurine and gamma-aminobutyric acid decreased after GFP labeling, whereas the concentrations of the other three metabolites increased. Three of these five metabolites (taurine, gamma-aminobutyric acid, and uracil) overlapped with the matching results from the dansyl standard compound library.

3.6. Metabolic Pathway Analysis. Metabolomic profiling of the 12 cell samples resulted in the identification of a total of 739 metabolites in the hPMSCs and $\mathrm{GFP}^{+} \mathrm{hPMSC}$. Eleven metabolites showed significant changes after labeling of hPMSCs with GFP. Five specific metabolites with significantly higher or lower concentrations in the $\mathrm{GFP}^{+} \mathrm{hPMSCs}$ than the hPMSCs were identified. The relevant metabolic pathways of these five metabolites were determined using MetaboAnalyst. Three of the identified metabolites (taurine, gamma-aminobutyric acid, and uracil) mapped to nine biologically relevant pathways (Table 2): taurine and hypotaurine metabolism (taurine); nitrogen metabolism (taurine); primary bile acid biosynthesis (taurine); alanine, aspartate, and glutamate metabolism (gamma-aminobutyric acid); beta-alanine metabolism (uracil, gamma-aminobutyric acid); butanoate metabolism (gamma-aminobutyric acid); arginine and proline metabolism (gamma-aminobutyric acid); pantothenate and CoA biosynthesis (uracil); and pyrimidine metabolism (uracil). A metabolomics view containing all of the matched pathways based on the pathway enrichment and pathway topology analyses with the Kyoto Encyclopedia of Genes and Genomes (KEGG; http://www.genome.jp/kegg/) pathway database is shown in Figure 4. A higher $p$ value and a higher impact value reflect the more relevant pathways affected by GFP labeling. Table 2 shows that these changed metabolites in the $\mathrm{GFP}^{+} \mathrm{hPMSC}$ are not likely to have a large impact on the metabolic pathways due to their relatively unimportant positions and limited hits (1 or 2). A simplified metabolic network of these nine biologically relevant pathways is presented in Figure 4, covering 305 compounds (see online Supplementary Table S2). Overall, pathway enrichment analysis indicated that GFP labeling did not induce significant changes in metabolism in hPMSCs.

\section{Discussion}

In stem cell therapy, fluorescent protein labeling technique provides a valuable molecular imaging tool to monitor and track the fate and function of cells by providing information on their survival, migration, proliferation, and differentiation status in recipient animals $[5,19,20]$. A recent review listed a few general requirements for the use of agents for cell tracking; for example, these labels must be nontoxic to cells in culture and animal models [21]. To utilize a tracking reagent, such as GFP, it is necessary to determine whether lentiviral transduction and expression of the GFP gene would affect the gene expression, metabolism, and biological characteristics of the cell. Our previous study of MSCs labeled with GFP found no effects on cell viability, proliferation rate, or differentiation capacity [5]. However, there have been no previous studies addressing whether GFP labeling has an impact on the metabolism of MSCs. Quantitative metabolomics techniques can be used to identify 


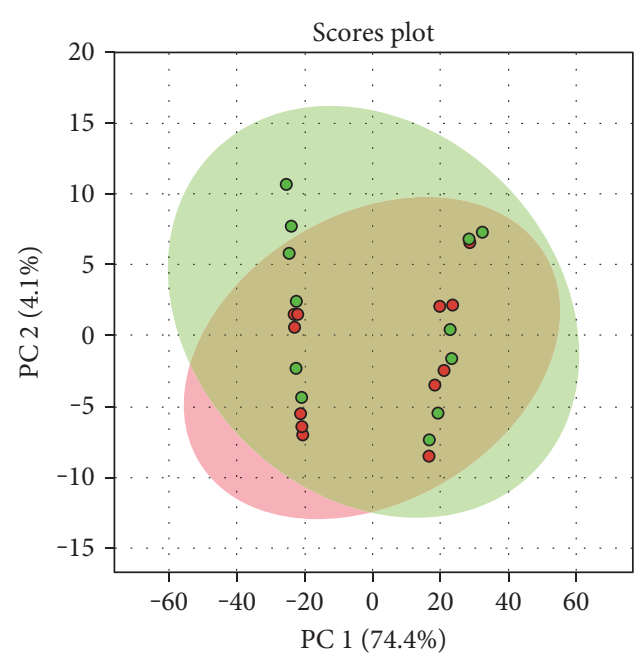

- $\mathrm{GFP}^{+} \mathrm{hPMSCs}(\mathrm{G})$

$\circ$ hPMSCs (P)

(a)

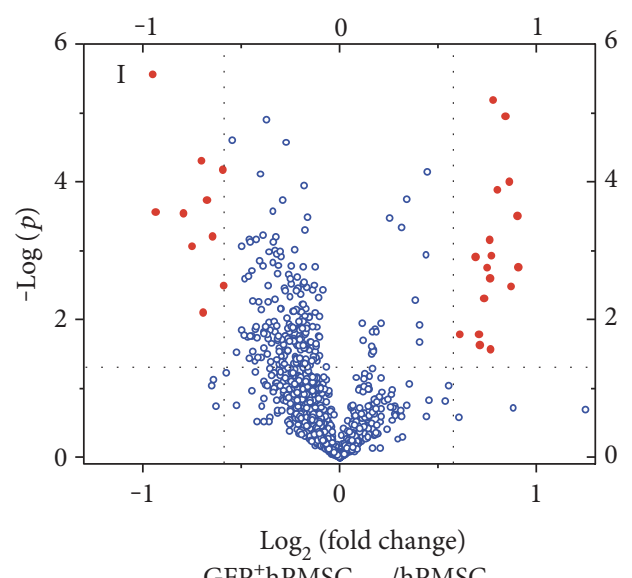

$\mathrm{GFP}^{+} \mathrm{hPMSC}_{\text {light }} / \mathrm{hPMSC}_{\text {heavy }}$

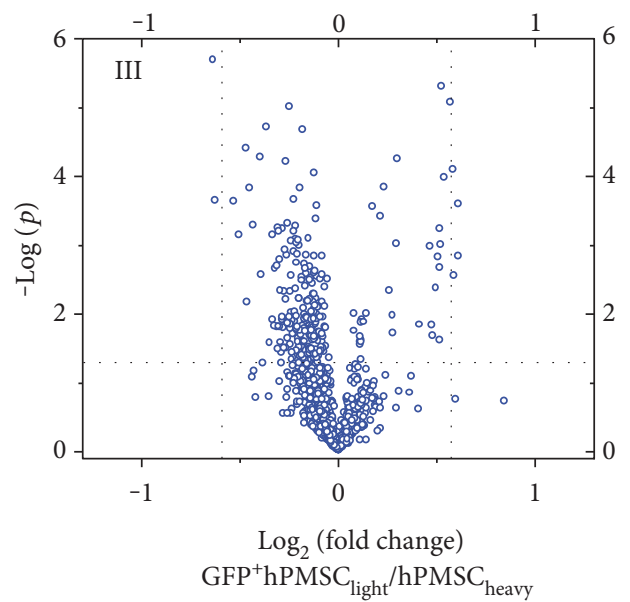

(c)

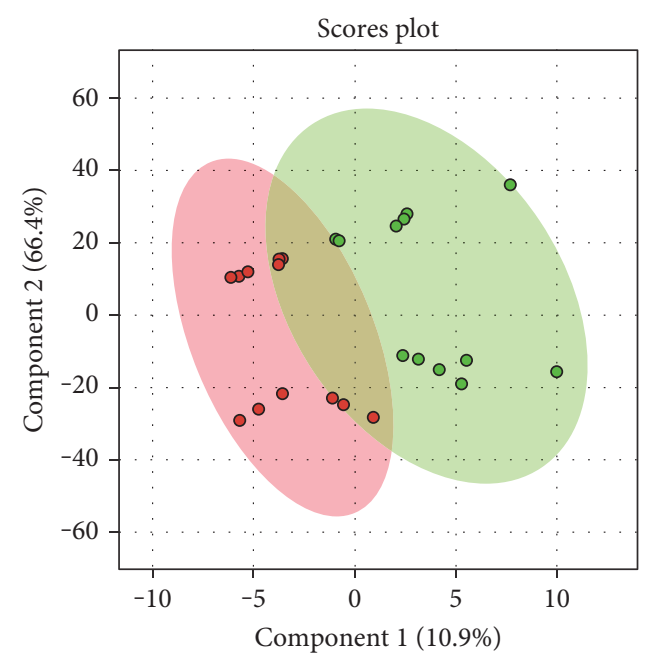

- $\mathrm{GFP}^{+} h \mathrm{hPSCs}(\mathrm{G})$

○ hPMSCs (P)

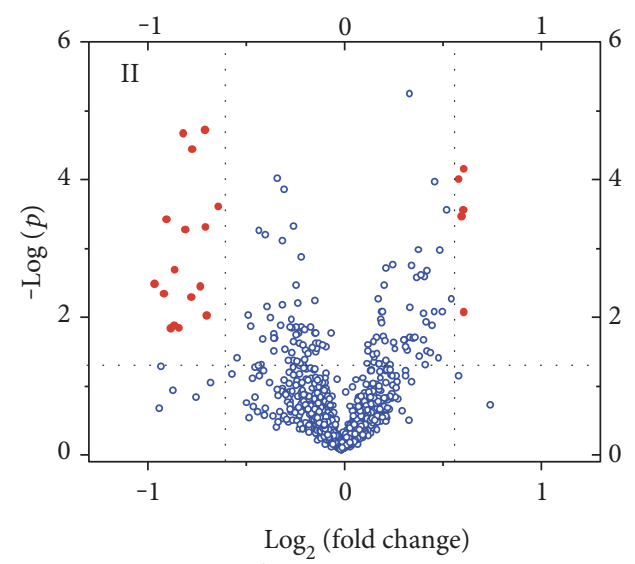

$\mathrm{GFP}^{+} \mathrm{hPMSC}_{\text {heavy }} / \mathrm{hPMSC}_{\text {light }}$

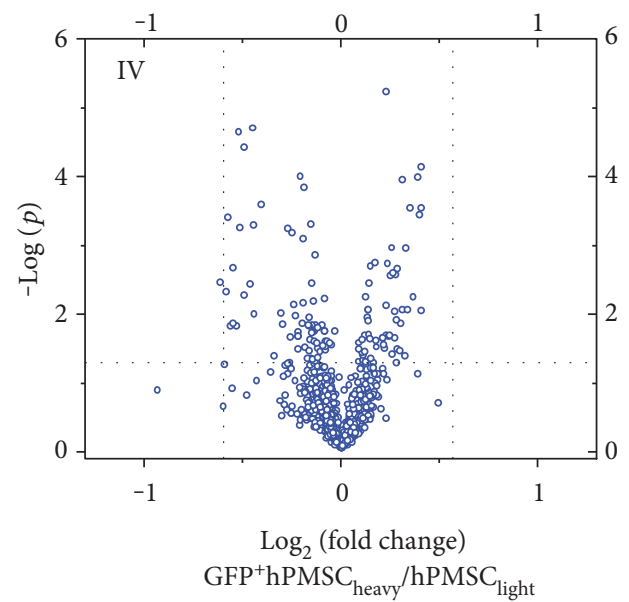

Figure 3: Metabolomics profiling. (a) Principal component analysis (PCA) plot of all of the data obtained from the LC-MS runs. The PCA score plot showed no separation between the hPMSCs and the GFP ${ }^{+}$hPMSCs. (b) Partial least squares discriminant analysis (PLS-DA) score plots of the hPMSCs and the GFP ${ }^{+}$hPMSCs. The PLS-DA score plot did not show a clear, valid separation between the two cell groups. (c) Volcano plots. Volcano plot analyses were used to determine the significant metabolites that separated the two groups (hPMSCs and $\mathrm{GFP}^{+} \mathrm{hPMSCs}$ ). The $x$-axis represents the $\log _{2}$ of the fold change (FC), which was plotted against the $-\log$ of the $p$ value. (c)I-II: $p<0.05$; data points with fold changes $>1.50$ or $<0.67$ are labeled red. (c)III-IV: $p<0.05$; data points with fold changes $>2$ or $<0.5$ are labeled red (no such data points were observed). 
TABLE 1: Candidate metabolites with significant changes between the hPMSC and GFP ${ }^{+} \mathrm{hPMSC}$ samples detected by isotope labeling liquid chromatography-mass spectrometry (CIL LC-MS) analysis.

(a)

\begin{tabular}{|c|c|c|c|c|c|c|c|}
\hline \multirow{2}{*}{$\begin{array}{l}\text { No. } \\
1\end{array}$} & \multicolumn{2}{|c|}{ Retention time $(\mathrm{s}) \mathrm{m} / \mathrm{z}$} & \multirow{2}{*}{$\begin{array}{c}{ }^{\Delta} \text { PG average } \\
1.89\end{array}$} & \multirow{2}{*}{$\begin{array}{c}{ }^{\dagger} \text { GP average } \\
0.47\end{array}$} & \multirow{2}{*}{$\begin{array}{c}\text { Candidate name } \\
\text { Taurine* }\end{array}$} & \multirow{2}{*}{$\begin{array}{c}\text { HMDB umber } \\
\text { HMDB00251 }\end{array}$} & \multirow{2}{*}{$\begin{array}{c}\text { Formula } \\
\mathrm{C}_{2} \mathrm{H}_{7} \mathrm{NO}_{3} \mathrm{~S}\end{array}$} \\
\hline & 200.90 & 125.0152 & & & & & \\
\hline 2 & 505.02 & 103.0634 & 2.02 & 0.55 & Gamma-aminobutyric acid* & HMDB00112 & $\mathrm{C}_{4} \mathrm{H}_{9} \mathrm{NO}_{2}$ \\
\hline 3 & 621.05 & 316.1400 & 0.69 & 1.58 & Dityrosine & HMDB06045 & $\mathrm{C}_{18} \mathrm{H}_{20} \mathrm{~N}_{2} \mathrm{O}_{6}$ \\
\hline 4 & 703.73 & 112.0278 & 0.42 & 2.39 & Uracil* $^{*}$ & HMDB00300 & $\mathrm{C}_{4} \mathrm{H}_{4} \mathrm{~N}_{2} \mathrm{O}_{2}$ \\
\hline 5 & 710.63 & 327.6295 & 0.57 & 1.83 & & & \\
\hline 6 & 780.23 & 319.6334 & 0.62 & 1.51 & & & \\
\hline 7 & 1046.74 & 380.5105 & 0.67 & 1.57 & & & \\
\hline 8 & 1127.03 & 243.4506 & 0.61 & 1.71 & & & \\
\hline 9 & 1156.70 & 87.1059 & 0.67 & 1.60 & & & \\
\hline 10 & 1252.74 & 101.1210 & 0.56 & 1.65 & & & \\
\hline 11 & 1336.96 & 115.1352 & 0.59 & 1.74 & DL-2-aminooctanoic acid & HMDB00991 & $\mathrm{C}_{8} \mathrm{H}_{17} \mathrm{NO}_{2}$ \\
\hline
\end{tabular}

(b)

\begin{tabular}{|c|c|c|c|c|c|c|c|c|c|c|c|c|}
\hline No. & $\begin{array}{c}{ }^{{ }^{\Delta}} \mathrm{PG1-1} \\
\text { ratio }\end{array}$ & $\begin{array}{c}{ }^{{ }^{\Delta}} \text { PG1-2 } \\
\text { ratio }\end{array}$ & $\begin{array}{c}{ }^{{ }^{\Delta}} \mathrm{PG1-3} \\
\text { ratio }\end{array}$ & $\begin{array}{c}{ }^{{ }^{\Delta}} \text { PG2-1 } \\
\text { ratio }\end{array}$ & $\begin{array}{c}{ }^{{ }^{\Delta}} \text { PG2-2 } \\
\text { ratio }\end{array}$ & $\begin{array}{c}{ }^{{ }^{\Delta}} \text { PG2-3 } \\
\text { ratio }\end{array}$ & $\begin{array}{c}{ }^{\dagger} \mathrm{GP} 1-1 \\
\text { ratio }\end{array}$ & $\begin{array}{c}{ }^{\dagger} \mathrm{GP} 1-2 \\
\text { ratio }\end{array}$ & $\begin{array}{c}{ }^{\dagger} \mathrm{GP} 1-3 \\
\text { ratio }\end{array}$ & $\begin{array}{c}{ }^{\dagger} \mathrm{GP} 2-1 \\
\text { ratio }\end{array}$ & $\begin{array}{c}{ }^{\dagger} \mathrm{GP} 2-2 \\
\text { ratio }\end{array}$ & $\begin{array}{c}{ }^{\dagger} \mathrm{GP} 2-3 \\
\text { ratio }\end{array}$ \\
\hline 1 & 3.09 & 1.33 & 2.80 & 1.58 & 1.47 & 1.07 & 0.21 & 0.56 & 0.34 & 0.47 & 0.57 & 0.65 \\
\hline 2 & 3.00 & 1.99 & 2.62 & 1.46 & 1.36 & 1.71 & 0.43 & 0.42 & 0.56 & 0.69 & 0.83 & 0.40 \\
\hline 3 & NA & NA & NA & 0.83 & 0.74 & 0.50 & NA & NA & NA & 1.33 & 1.78 & 1.64 \\
\hline 4 & NA & NA & NA & 0.23 & 0.56 & 0.46 & NA & NA & NA & 3.92 & 1.47 & 1.77 \\
\hline 5 & NA & NA & NA & 0.76 & 0.34 & 0.62 & NA & NA & NA & 1.03 & 3.00 & 1.45 \\
\hline 6 & NA & NA & NA & 0.95 & 0.36 & 0.54 & NA & NA & NA & 0.75 & 2.47 & 1.29 \\
\hline 7 & NA & NA & NA & 0.89 & 0.42 & 0.69 & NA & NA & NA & 1.19 & 2.28 & 1.24 \\
\hline 8 & NA & NA & NA & 0.71 & 0.69 & 0.43 & NA & NA & NA & 1.52 & 1.54 & 2.09 \\
\hline 9 & NA & NA & NA & 0.98 & 0.44 & 0.59 & NA & NA & NA & 0.83 & 2.12 & 1.84 \\
\hline 10 & NA & NA & NA & 0.77 & 0.45 & 0.46 & NA & NA & NA & 1.15 & 2.09 & 1.71 \\
\hline 11 & NA & NA & NA & 0.82 & 0.37 & 0.58 & NA & NA & NA & 0.94 & 2.65 & 1.64 \\
\hline
\end{tabular}

${ }^{*}$ Metabolites that overlapped with the matching result from the dansyl standard compound library. ${ }^{\Delta \dagger}$ List of 11 significant metabolites with correlated responses from the hPMSCs $(\mathrm{P})$ and the $\mathrm{GFP}^{+} \mathrm{hPMSCs}(\mathrm{G})$ with the different extraction methods $(1,2)$ in triplicate $(-1$ to -3$)$. HMDB: human metabolome database.

TAble 2: Pathway analysis of the three identified metabolites (taurine, gamma-aminobutyric acid, and uracil) using MetaboAnalyst. These metabolites were related to nine metabolic pathways.

\begin{tabular}{lcccccc}
\hline No. & Metabolic pathway & Total & Expected & Hits & FDR & Impact \\
\hline 1 & Beta-alanine metabolism & 28 & 0.034898 & 2 & 0.031104 & 0 \\
2 & Taurine and hypotaurine metabolism & 20 & 0.024927 & 1 & 0.65401 & 0.33094 \\
3 & Alanine, aspartate, and glutamate metabolism & 24 & 0.029913 & 1 & 0.65401 & 0.10256 \\
4 & Pantothenate and CoA biosynthesis & 27 & 0.033652 & 1 & 0.65401 & 0 \\
5 & Nitrogen metabolism & 39 & 0.048608 & 1 & 0.65401 & 0 \\
6 & Butanoate metabolism & 40 & 0.049855 & 1 & 0.65401 & 0.01067 \\
7 & Primary bile acid biosynthesis & 47 & 0.058579 & 1 & 0.65676 & 0.00822 \\
8 & Pyrimidine metabolism & 60 & 0.074782 & 1 & 0.72963 & 0.07132 \\
9 & Arginine and proline metabolism & 77 & 0.09597 & 1 & 0.8264 & 0.01905 \\
\hline
\end{tabular}

changes in metabolite contents, which indicate host cellular responses to gene transfection.

To examine the cellular metabolic changes, it is important to achieve high coverage of metabolites and cover as many pathways as possible. In this regard, the successful extraction of metabolites from cells is a critical step [22]. There have been many in-depth studies on sample preparation for metabolomics analyses of adherent mammalian cells 


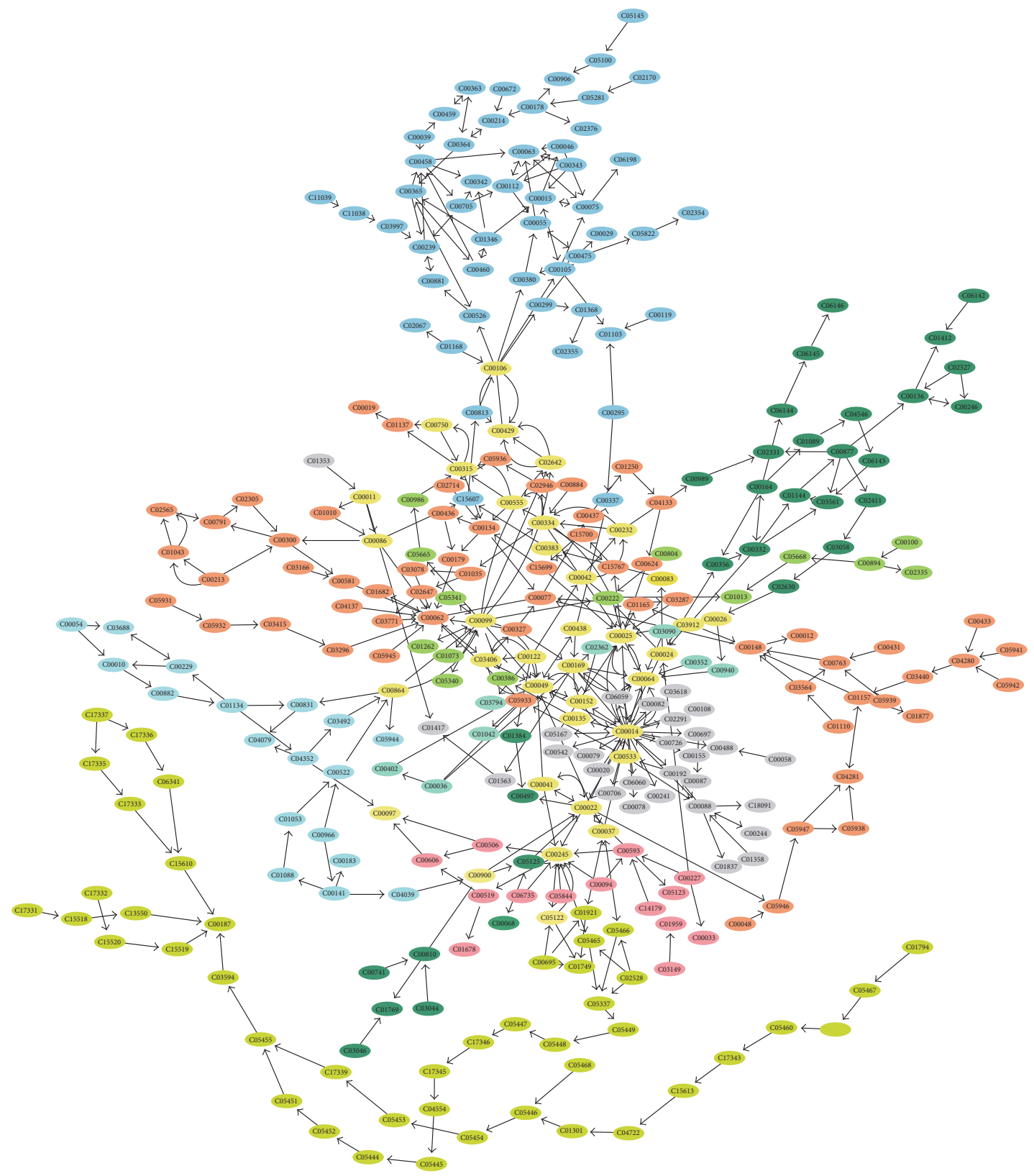

Nine metabolic pathways

$$
\begin{aligned}
& \text { Primary bile acid } \\
& \text { biosynthesis } \\
& \text { Pyrimidine metabolism } \\
& \text { Alanine, aspartate, and } \\
& \text { glutamate metabolism } \\
& \text { Taurine and hypotaurine } \\
& \text { metabolism }
\end{aligned}
$$

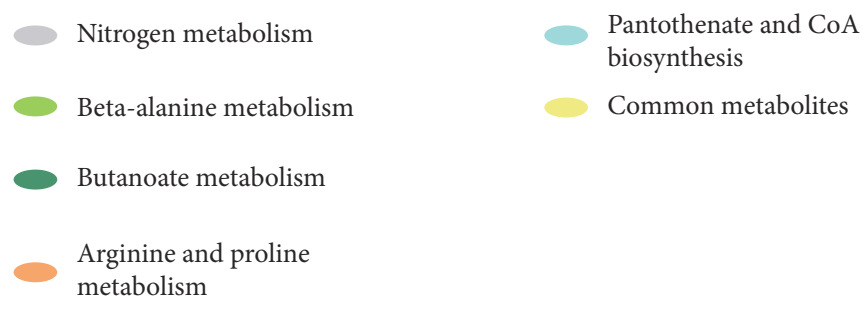

Figure 4: Kyoto Encyclopedia of Genes and Genomes (KEGG) datasets of the nine metabolic pathways were analyzed and visualized using Cytoscape 3.4.0 on MetScape. The network was integrated with 305 compounds from the nine metabolic pathways. A function-guided layout was used to organize the highly connected network. The different metabolic pathways are mapped to different node colors. The corresponding compound of each KEGG ID is shown in online Supplementary Table S2. The yellow nodes represent the common metabolites involved in the different metabolic pathways. The red asterisk-marked node represents the three identified metabolites (taurine, gamma-aminobutyric acid, and uracil). 
[11-13]. Lorenz et al. reported a convenient and adaptable workflow for the preparation of adherent mammalian cell samples [11]. This workflow was rapid and convenient and provided good sensitivity for detecting a variety of metabolites. The procedure included a rapid water rinse, $\mathrm{LN}_{2}$ quenching, and a single-step extraction. For the extraction step, the authors initially evaluated ethanol (EtOH), ACN, $\mathrm{MeOH}$, and $9 / 1 \mathrm{MeOH} / \mathrm{CHCl}_{3}$ for their ability to extract and stabilize metabolites. Overall, the $9 / 1 \mathrm{MeOH} / \mathrm{CHCl}_{3}$ solvent provided superior metabolite recovery and extract stability. In another study, $\mathrm{Wu}$ and $\mathrm{Li}$ evaluated the performances of three extraction solvent systems $(1 / 1 \mathrm{MeOH} /$ $\mathrm{H}_{2} \mathrm{O}, 1 / 1 \mathrm{ACN} / \mathrm{H}_{2} \mathrm{O}$, and $2 / 2 / 1 \mathrm{MeOH} / \mathrm{ACN} / \mathrm{H}_{2} \mathrm{O}$ ) [12]. They found that the $1 / 1 \mathrm{MeOH} / \mathrm{H}_{2} \mathrm{O}$ solvent system performed significantly better than the $\mathrm{ACN}$ or $\mathrm{MeOH} / \mathrm{ACN}$ systems in terms of reproducibility and relative extraction efficiency. Based on these findings and given that different solvent mixtures may have different metabolite extraction and solubility properties, both of these reported sample preparation methods were applied in this study to extract the metabolites of hPMSCs before and after GFP gene transfection with $9 / 1 \mathrm{MeOH} / \mathrm{CHCl}_{3}$ and $1 / 1 \mathrm{MeOH} / \mathrm{H}_{2} \mathrm{O}$ as extraction solvents. Our results showed that the $1 / 1 \mathrm{MeOH} / \mathrm{H}_{2} \mathrm{O}$ solvent extracted more metabolites with higher peak intensities than the $9 / 1 \mathrm{MeOH} / \mathrm{CHCl}_{3}$ solvent Even though 327 metabolites were detected in both methods, hundreds of unique metabolites were extracted from each of the two methods, indicating that metabolites with different chemical and physical properties could be extracted from different solvent systems. Integration of the metabolites extracted from both methods represented a better coverage of the metabolome and provided a more comprehensive view of the changes that happened in cellular metabolism.

In addition to achieving efficient metabolite extraction, metabolite detection is also critical to achieve high metabolome coverage. Among the various analytical platforms used for metabolome analysis, LC-MS is the most widely used [23]. Recently, we developed a chemical isotope labeling technique (CIL LC-MS) that is suitable for profiling metabolites containing primary or secondary amines or phenol groups [24]. For CIL LC-MS, ${ }^{12} \mathrm{C} /{ }^{13} \mathrm{C}$-dansyl reagent is added to label the metabolites before loading the samples into an LC-MS system. These isotope-labeled metabolites are then detected as peak pairs in the mass spectra, and the peak intensity ratio is used for metabolic quantification. Due to the chemical properties of the dansyl group, this technique provides a $10-1000$-fold increase in detection sensitivity and is capable of separating polar and ionic metabolites after labeling, enabling the detection of thousands of metabolites using one-dimensional LC-MS. The use of isotope labeling of metabolites from two different groups of cells also provides much-improved quantification accuracy as the light/heavy metabolite ion pairs always experience the same matrix when detected in the mass spectrometer [25]. Chemical isotope labeling has been successfully applied to metabolomics studies of various biological samples to discover potential biomarkers and investigate cellular metabolomics [24, 26, 27]. In this study, the differential ${ }^{12} \mathrm{C} /{ }^{13} \mathrm{C}$-dansylation labeling strategy allowed 1151 metabolite peak pairs to be detected from adherent mammalian cells. In particular, the forward-andreverse labeling strategy we have applied allowed us to minimize the impact of system errors and greatly enhanced the confidence of both the identification and the quantification results.

Multivariate statistical analyses of the quantitative data using PCA and PLS-DA showed poor separation between the hPMSCs and GFP ${ }^{+}$hPMSCs. Volcano plot analyses also demonstrated that, for thresholds of $\mathrm{FC}>1.5$ or $\mathrm{FC}<0.67$ and $p<0.05$, the majority of the metabolites $(98.0 \%)$ were not significantly changed when the hPMSCs were labeled with GFP. No metabolites showed significant changes for thresholds of FC $>2$ or FC $<0.5$ and $p<0.05$. Hierarchical clustering and heat maps also revealed that the hPMSCs and the $\mathrm{GFP}^{+} \mathrm{hPMSC}$ did not show markedly distinct metabolite patterns. In addition, among the 1151 metabolites detected, only 11 showed significant changes after GFP labeling, and five of these metabolites were identified. Metabolic pathway analysis indicated that three of these metabolites (taurine, uracil, and gamma-aminobutyric acid) were involved in nine pathways, with only one or two hits. And two other metabolites, DL-2-aminooctanoic acid and dityrosine, were not in the KEGG pathway database. However, due to their relatively nonessential positions, these changes were unlikely to markedly affect the metabolic pathways.

\section{Conclusions}

In this study, as well as in our previous studies, we report that the labeling of MSCs with GFP is safe and does not affect the metabolism or functions of MSCs. This is the first reported study of the metabolism of MSCs and $\mathrm{GFP}^{+} \mathrm{hPMSCs}$ using an isotope labeling LC-MS method, which provides a new method to examine the metabolism of stem cells for cell-based therapies.

\section{Abbreviations}

MSCs:

hPMSCs:

GFP: $\quad$ Green fluorescent protein

UHPLC-Q/TOF-MS: Ultra-high performance liquid chromatography-quadrupole/time-offlight mass spectrometry

CIL LC-MS: $\quad$ Chemical isotope labeling liquid chromatography-mass spectrometry

PCA:

PLS-DA: Principal component analysis

HMDB:

BPI:

$\mathrm{LN}_{2}$ :

$\mathrm{MeOH}$ :

$\mathrm{CHCl}_{3}$ :

ACN:

FA:

Partial least squares discriminant analysis

Human metabolome database

Based peak intensity

Liquid nitrogen

Methyl alcohol

Trichloromethane

Acetonitrile

Formic acid

RT:

Retention time. 


\section{Additional Points}

Highlights. (i) ${ }^{13} \mathrm{C} /{ }^{12} \mathrm{C}$-dansyl labeling provides $10-1000$-fold increases in sensitivity for relative quantification of individual metabolites. (ii) A total of 1151 peak pairs or metabolites were detected. Only 11 metabolites showed significant changes after labeling of human placental mesenchymal stem cells (hPMSCs) with green fluorescent protein (GFP). Pathway analyses using MetaboAnalyst indicated that GFP labeling did not induce significant changes in hPMSC metabolism. (iii) hPMSCs and $\mathrm{GFP}^{+}$hPMSCs did not show markedly distinct metabolite patterns, as determined based on the results of principal component analysis, partial least squares discriminant analysis, volcano plots, and hierarchical clustering analysis.

\section{Conflicts of Interest}

No competing financial interests exist in the subject matter or materials discussed in the presented work.

\section{Authors' Contributions}

Hongcui Cao, Jinfeng Yang, and Nan Wang are responsible for the conception and design. Cell preparation was done by Qiaoling Pan, Xiaotian Dong, and Xiaowei Shi. Nan Wang, Deying Chen, Dan Wang, Jiong Yu, and Jingqi Liu are responsible for the analysis and interpretation of data. Hongcui Cao, Jinfeng Yang, Nan Wang, and Deying Chen drafted the manuscript. Liang Li and Lanjuan Li supervised the study. All authors critically revised the manuscript for intellectual content. Jinfeng Yang and Nan Wang contributed equally to this work.

\section{Acknowledgments}

This study was supported by Stem Cell and Translational Research, National Key Research and Development Program of China (no. 2016YFA0101001) and the National Natural Science Foundation of China (no. 81471794).

\section{References}

[1] P. Bianco, X. Cao, P. S. Frenette et al., "The meaning, the sense and the significance: translating the science of mesenchymal stem cells into medicine," Nature Medicine, vol. 19, pp. 3542, 2013.

[2] C. Nombela-Arrieta, J. Ritz, and L. E. Silberstein, "The elusive nature and function of mesenchymal stem cells," Nature Reviews Molecular Cell Biology, vol. 12, pp. 126-131, 2011.

[3] S. Wang and T. Hazelrigg, "Implications for bcd mRNA localization from spatial distribution of exu protein in Drosophila oogenesis," Nature, vol. 369, pp. 400-403, 1994.

[4] C. J. Daly and J. C. McGrath, "Fluorescent ligands, antibodies, and proteins for the study of receptors," Pharmacology \& Therapeutics, vol. 100, pp. 101-118, 2003.

[5] J. Yu, X. Su, C. Zhu et al., "GFP labeling and hepatic differentiation potential of human placenta-derived mesenchymal stem cells," Cellular Physiology and Biochemistry, vol. 35, pp. 2299-2308, 2015.
[6] A. Nordstrom and R. Lewensohn, "Metabolomics: moving to the clinic," Journal of Neuroimmune Pharmacology, vol. 5, pp. 4-17, 2010.

[7] S. P. Putri, Y. Nakayama, F. Matsuda et al., "Current metabolomics: practical applications," Journal of Bioscience and Bioengineering, vol. 115, pp. 579-589, 2013.

[8] L. E. McNamara, T. Sjostrom, R. M. Meek et al., "Metabolomics: a valuable tool for stem cell monitoring in regenerative medicine," Journal of the Royal Society Interface, vol. 9, pp. 1713-1724, 2012.

[9] A. Mastrangelo, M. I. Panadero, L. M. Perez et al., "New insight on obesity and adipose-derived stem cells using comprehensive metabolomics," Biochemical Journal, vol. 473, pp. 21872203, 2016.

[10] H. Cao, J. Yang, J. Yu et al., “Therapeutic potential of transplanted placental mesenchymal stem cells in treating Chinese miniature pigs with acute liver failure," BMC Medicine, vol. 10, p. 56, 2012.

[11] M. A. Lorenz, C. F. Burant, and R. T. Kennedy, "Reducing time and increasing sensitivity in sample preparation for adherent mammalian cell metabolomics," Analytical Chemistry, vol. 83, pp. 3406-3414, 2011.

[12] Y. Wu and L. Li, "Development of isotope labeling liquid chromatography-mass spectrometry for metabolic profiling of bacterial cells and its application for bacterial differentiation," Analytical Chemistry, vol. 85, pp. 5755-5763, 2013.

[13] G. Martano, N. Delmotte, P. Kiefer et al., "Fast sampling method for mammalian cell metabolic analyses using liquid chromatography-mass spectrometry," Nature Protocols, vol. 10, pp. 1-11, 2015.

[14] R. Zhou and L. Li, "Effects of sample injection amount and time-of-flight mass spectrometric detection dynamic range on metabolome analysis by high-performance chemical isotope labeling LC-MS," Journal of Proteomics, vol. 118, pp. 130-139, 2015.

[15] T. Huan and L. Li, "Counting missing values in a metaboliteintensity data set for measuring the analytical performance of a metabolomics platform," Analytical Chemistry, vol. 87, pp. 1306-1313, 2015.

[16] P. Shannon, A. Markiel, O. Ozier et al., "Cytoscape: a software environment for integrated models of biomolecular interaction networks," Genome Research, vol. 13, pp. 2498-2504, 2003.

[17] Y. Wu, F. Streijger, Y. Wang et al., "Parallel metabolomic profiling of cerebrospinal fluid and serum for identifying biomarkers of injury severity after acute human spinal cord injury," Scientific Reports, vol. 6, article 38718, 2016.

[18] L. Li, R. Li, J. Zhou et al., "MyCompoundID: using an evidence-based metabolome library for metabolite identification," Analytical Chemistry, vol. 85, pp. 3401-3408, 2013.

[19] T. Arai, T. Kofidis, J. W. Bulte et al., "Dual in vivo magnetic resonance evaluation of magnetically labeled mouse embryonic stem cells and cardiac function at 1.5 t," Magnetic Resonance in Medicine, vol. 55, pp. 203-209, 2006.

[20] H. Kimura, Y. Hayashi-Takanaka, T. J. Stasevich, and Y. Sato, "Visualizing posttranslational and epigenetic modifications of endogenous proteins in vivo," Histochemistry and Cell Biology, vol. 144, pp. 101-109, 2015.

[21] J. C. Wu, I. Y. Chen, G. Sundaresan et al., "Molecular imaging of cardiac cell transplantation in living animals using optical bioluminescence and positron emission tomography," Circulation, vol. 108, pp. 1302-1305, 2003. 
[22] Z. Leon, J. C. Garcia-Canaveras, M. T. Donato, and A. Lahoz, "Mammalian cell metabolomics: experimental design and sample preparation," Electrophoresis, vol. 34, pp. 2762-2775, 2013.

[23] Z. Z. Fang and F. J. Gonzalez, "LC-MS-based metabolomics: an update," Archives of Toxicology, vol. 88, pp. 1491-1502, 2014.

[24] K. Guo, F. Bamforth, and L. Li, "Qualitative metabolome analysis of human cerebrospinal fluid by ${ }^{13} \mathrm{C}-{ }^{12} \mathrm{C}$-isotope dansylation labeling combined with liquid chromatography Fourier transform ion cyclotron resonance mass spectrometry," Journal of the American Society for Mass Spectrometry, vol. 22, pp. 339-347, 2011.

[25] G. M. Mackay, L. Zheng, N. J. van den Broek, and E. Gottlieb, "Analysis of cell metabolism using LC-MS and isotope tracers," Methods in Enzymology, vol. 561, pp. 171-196, 2015.

[26] E. Gimenez, V. Sanz-Nebot, and A. Rizzi, "Relative quantitation of glycosylation variants by stable isotope labeling of enzymatically released $\mathrm{N}$-glycans using $\left[{ }^{12} \mathrm{C}\right] /\left[{ }^{13} \mathrm{C}\right]$ aniline and ZIC-HILIC-ESI-TOF-MS," Analytical and Bioanalytical Chemistry, vol. 405, pp. 7307-7319, 2013.

[27] A. Chokkathukalam, D. H. Kim, M. P. Barrett, R. Breitling, and D. J. Creek, "Stable isotope-labeling studies in metabolomics: new insights into structure and dynamics of metabolic networks," Bioanalysis, vol. 6, pp. 511-524, 2014. 

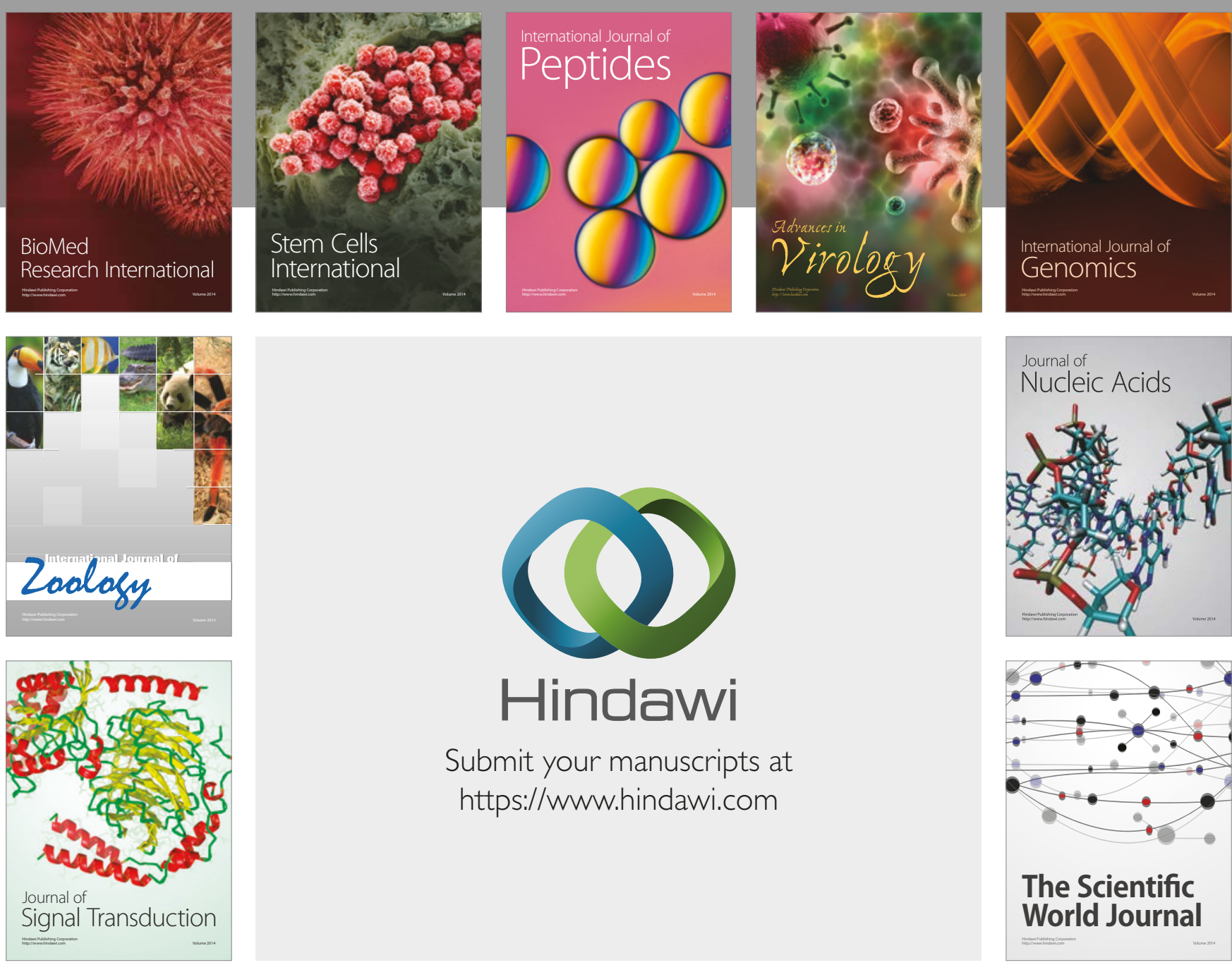

Submit your manuscripts at

https://www.hindawi.com
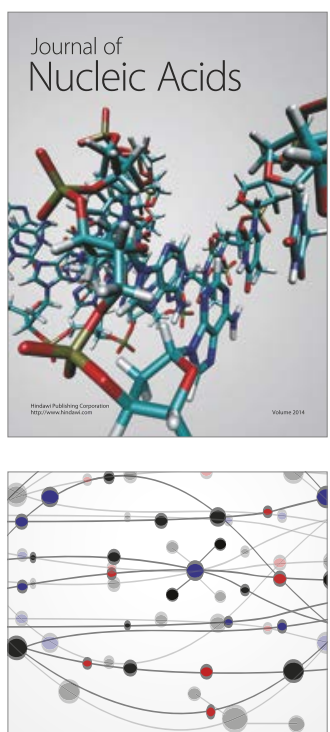

The Scientific World Journal

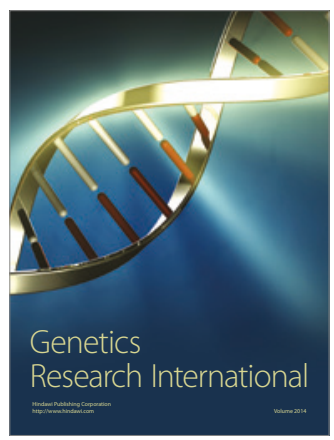

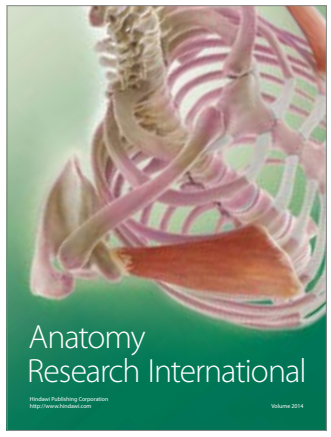

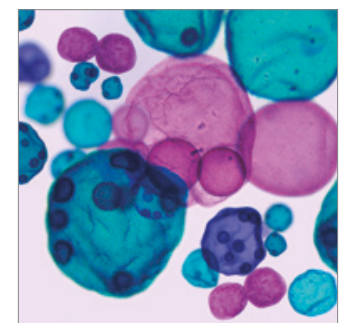

International Journal of Microbiology
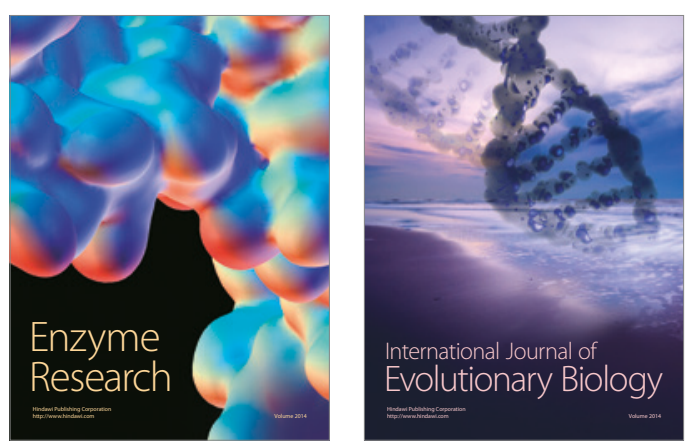
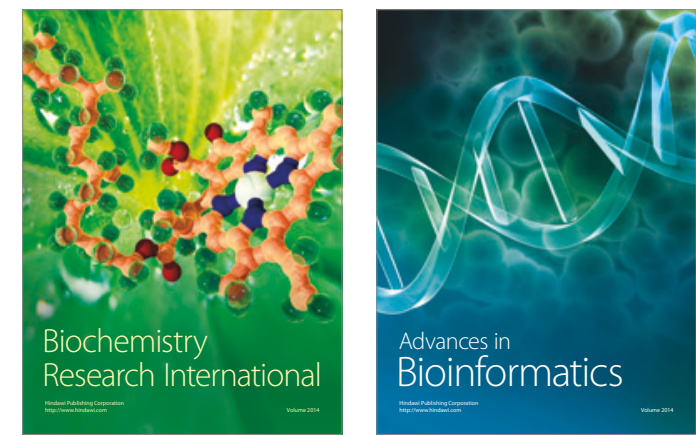

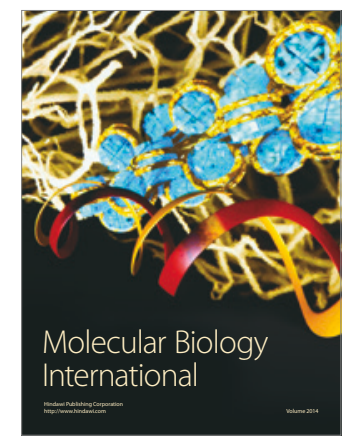

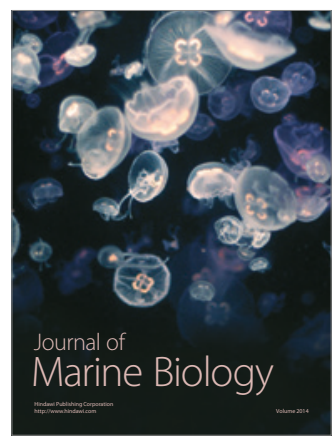

